\title{
Comparing accuracy of denture bases fabricated by injection molding, CAD/CAM milling, and rapid prototyping method
}

\author{
Suji Lee ${ }^{1 a}$, Seoung-Jin Hong ${ }^{2 a}$, Janghyun Paek ${ }^{3}$, Ahran Pae ${ }^{3}$, Kung-Rock Kwon ${ }^{3}$, Kwantae Noh ${ }^{3 *}$ \\ ${ }^{1}$ Private Practice, Seoul, Republic of Korea \\ ${ }^{2}$ Department of Prosthodontics, Kyung Hee University Dental Hospital, Seoul, Republic of Korea \\ ${ }^{3}$ Department of Prosthodontics, School of Dentistry, Kyung Hee University, Seoul, Republic of Korea
}

\begin{abstract}
PURPOSE. The accuracy of denture bases was compared among injection molding, milling, and rapid prototyping (RP) fabricating method. MATERIALS AND METHODS. The maxillary edentulous master cast was fabricated and round shaped four notches were formed. The cast was duplicated to ten casts and scanned. In the injection molding method, designed denture bases were milled from a wax block and fabricated using SR Ivocap injection system. Denture bases were milled from a pre-polymerized block in the milling method. In the RP method, denture bases were printed and post-cured. The intaglio surface of the base was scanned and surface matching software was used to measure inaccuracy. Measurements were performed between four notches and two points in the mid-palatal suture to evaluate inaccuracy. The palatine rugae resolution was evaluated. One-way analysis of variance was used for statistical analysis at $\alpha=.05$. RESULTS. No statistically significant differences in distances among four notches $(P>.05)$. The accuracy of the injection molding method was lower than those of the other methods in two points of the mid-palatal suture significantly $(P<.05)$. The degree of palatine rugae resolution was significantly higher in the injection molding method than that in other methods $(P<.05)$. CONCLUSION. The overall accuracy of the denture base is higher in milling and RP method than the injection molding method. The degree of fine reproducibility is higher in the injection molding method than the milling or RP method. [J Adv
\end{abstract}

Prosthodont 2019;11:55-64]

KEYWORDS: Computer-aided design and computer-aided manufacturing (CAD/CAM); Denture base; Milling; 3D printing; Digital light processing

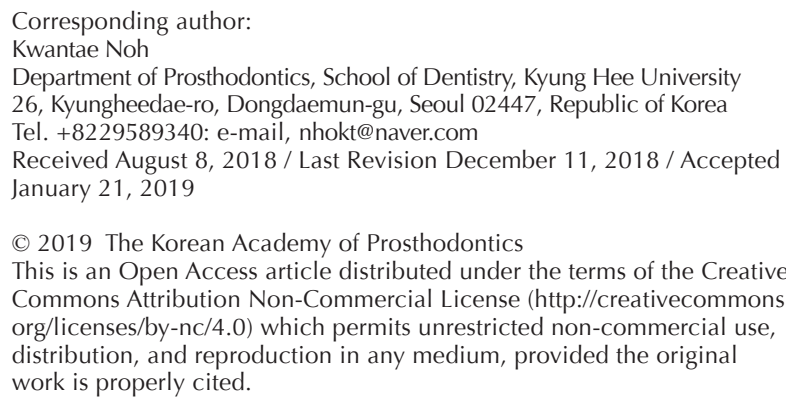
Commons Attribution Non-Commercial License (http://creativecommons. org/licenses/by-nc/4.0) which permits unrestricted non-commercial use, distribution, and reproduction in any medium, provided the original work is properly cited.

a These authors contributed equally to this work.

This work was supported by the National Research Foundation of Korea (NRF) grant funded by the Korea government (MSIT) (No. NRF-2017R1C1B5076904) and a grant from Kyung Hee University in 2015 (KHU-20150841).

\section{INTRODUCTION}

Heat-polymerization polymethyl methacrylate (PMMA) denture base deforms during polymerization, cooling, and finishing, resulting in linear deformation of $0.45-0.9 \%{ }^{1,2}$ The fit between denture base and mucosal tissue is important for the retention of complete denture. Deformation and shrinkage affect the retention, stabilization, and support of complete denture, thus affecting patients' satisfaction and quality of life. ${ }^{3}$

To fabricate complete denture, conventional method was developed approximately 80 years ago. ${ }^{4}$ To overcome and compensate for such polymerization shrinkage, many studies have been performed, including denture base materials, fabrication methods, and clinical protocols such as laboratory re-mounting procedure. ${ }^{5-7}$ To solve dimensional inaccuracy of the compression molding method, Pryor has 
attempted a pressure injection molding method in which the un-polymerized acrylic resin was injected into the mold. ${ }^{8}$ In the 1970s, Ivoclar (Schaan, Liechtenstein) introduced a pressure injection molding method with improved resin. Since then, many companies have introduced pressure injection molding methods. Such methods are now commonly used in the fabrication of complete dentures. ${ }^{9}$ However, most of these studies and methods have been conducted within the framework of traditional methods.

In the last 20 years, computer-aided design (CAD) and computer-aided manufacturing (CAM) technology has been broadly used in the dental field for manufacturing various prostheses due to its advantages such as increased efficiency, automaticity, and accuracy of the entire treatment flow. Fabrication of complete denture by CAD/CAM procedure has been realized and progressed by the development of digital devices and CAD software. ${ }^{10-12}$ Maeda et al. have reported the first scientific report describing the CAD/ CAM procedure for fabricating a complete denture. ${ }^{13}$ When manufacturing a complete denture using digital workflow, the process begins with digital scanning of the edentulous arch, including challenging areas for the intraoral scanner device to scan, which are movable areas such as non-keratinized tissue and smooth surfaces covered with saliva. ${ }^{13,14}$ Many researches have been inclined to scan the edentulous master cast using an extraoral scanner. ${ }^{15}$ After digitalization of conventional impression, complete denture can be designed using CAD software. The fabricating of the denture base or the entire complete denture can be processed by either milling or rapid prototyping $(\mathrm{RP})$ with a $3 \mathrm{D}$ printer.

The base of the complete denture is essential for its retention. Clinical treatment results are greatly influenced by the accuracy of the base. ${ }^{3}$ Computer numerical control (CNC) machines have been used in many studies to fabricate high accuracy denture bases. ${ }^{11,16,17}$ Avadent and Dentca, the first two companies that commercialized CAD/CAM dentures, were taking different approaches for fabricating method, milling, and 3D printing. These technological advancements have provided competent methods to facilitate fast and accurate fabrication of bases. Goodacre et al. ${ }^{18}$ have compared the fit of the base made by heat compres- sion, fluid resin, injection molding, and CAD/CAM milling methods and found that the CAD/CAM milling method is accurate and reproducible. McLaughlin et al. ${ }^{19}$ have compared the deformation of denture bases manufactured by three methods: compression molding, injection molding, and CAD/CAM milling. The influence on shrinkage by arch shape and palate depth was also studied. They found that well and equally fitted dentures were produced by injection molding and CAD/CAM milling methods, and both methods showed better fit accuracy than compression molding method. ${ }^{19}$ Many other studies have also compared the accuracy of bases fabricated by conventional manufacturing methods and milling methods. However, research on the accuracy of the base made by RP method using 3D printers is insufficient.

Therefore, the aim of this study was to evaluate the accuracy and surface resolution of denture bases fabricated by three methods: injection molding, milling, and RP using surface matching software. The null hypothesis was that there would be no difference in the accuracy or resolution among denture bases fabricated with the three methods.

\section{MATERIALS AND METHODS}

Thirty maxillary complete denture bases (10 denture bases for each group, 3 groups) were fabricated and evaluated (Fig. 1). For the master cast of this study, medium palate depth (12 mm palate depth) was selected according to palate depth classification of edentulous patient described by Johnson et al. ${ }^{20}$ In the master cast, right and left points crossed anterior alveolar ridge and posterior alveolar ridges and points on posterior alveolar ridge were $15 \mathrm{~mm}$ in distance, parallel to the midpalatal suture from right and left hamular notches that were engraved $1 \mathrm{~mm}$ diameter using No. 2 carbide round bur (Burstar, Alphadent, Goyang, Korea) (Fig. 2). The master cast with index notches was duplicated and ten casts were fabricated using silicone (Deguform, Dentsply international, Hanau, Germany) and type IV gypsum (GC FujiRock, GC Europe, Leuven, Belgium) according to the manufacturers' instructions. Using a record base and a record rim, casts were mounted on a semi-adjustable

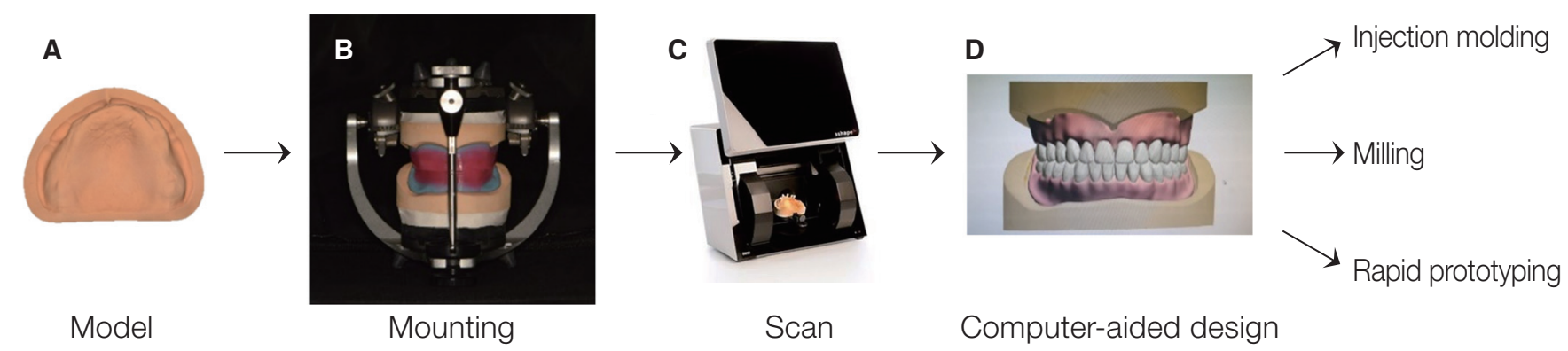

Fig. 1. Schematic illustration of denture base fabrication. 


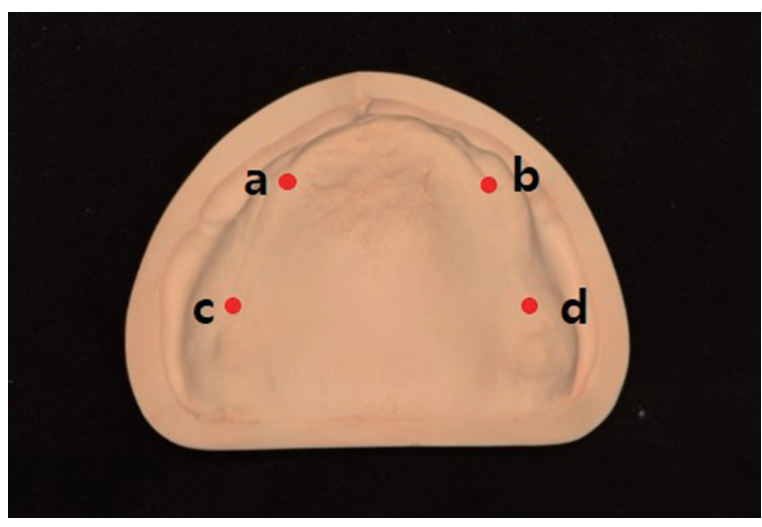

Fig. 2. Points crossing the anterior alveolar ridge and posterior alveolar ridges (right: a, left: b) and points on posterior alveolar ridge from $15 \mathrm{~mm}$ distance parallel to the midpalatal suture from right and left hamular notches (right: c, left: d).

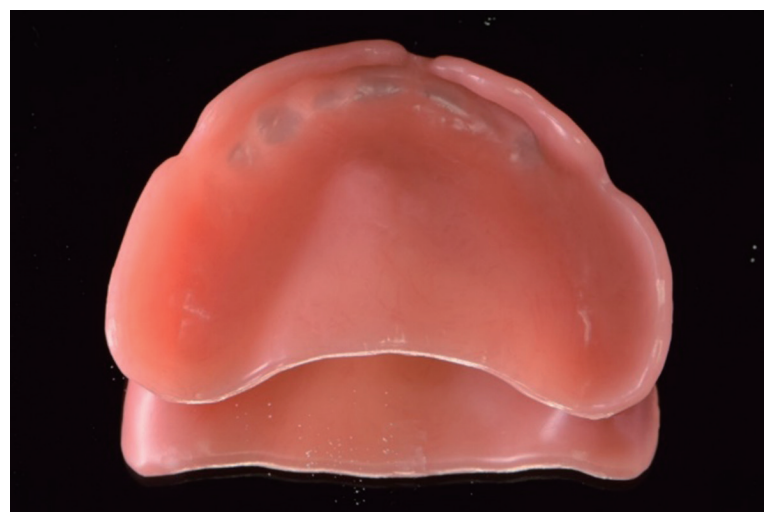

Fig. 4. Denture base fabricated by milling method.
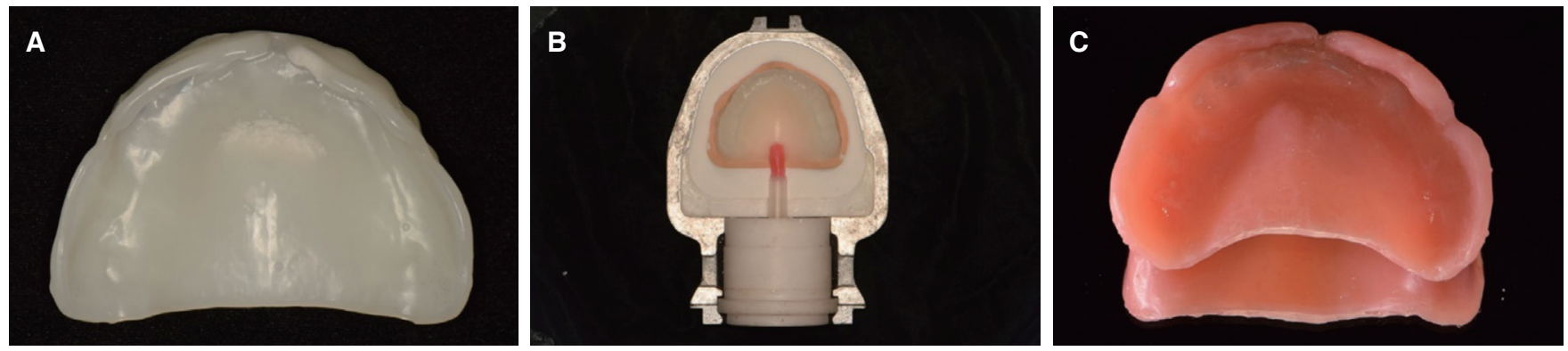

Fig. 3. Injection molding method process. (A) Denture base fabricated from wax block by milling, (B) Investment of denture base, (C) Fabricated denture base.

articulator based on the fact that Korean edentulous patients had an average value of $23 \mathrm{~mm}$ from anterior border to the occlusal surface for the maxilla and $18 \mathrm{~mm}$ for the mandible. After scanning mounted casts with a record base and a record rim using a model scanner (D800, 3Shape A/S., Copenhagen, Denmark), the denture base was designed, including denture polished surface and intaglio surface. Artificial teeth alignment was performed using CAD software (3shape dental designer, 3Shape A/S, Copenhagen, Denmark). The denture base of the palate was designed in standardized form (2 $\mathrm{mm}$ in thickness). Denture bases for the three groups were fabricated based on same standard tessellation language (STL) files designed in the CAD software (Fig. 1).

In the injection molding method group, wax block (Mazic wax, Vericom, Daejeon, Korea) was milled based on the STL file designed in the CAD software and the base was fabricated using an Ivocap injection system (Ivoclar Vivadent AG, Schaan, Liechtenstein) with PMMA resin (SR-Ivocap high impact, Ivoclar Vivadent AG) (Fig. 3). For the milling method group, denture bases were fabricated with CAD/ CAM milling using CAM software (HyperDent, FOLLOW-
ME! Technology, Munich, Germany) and a 5-axis milling machine (ARUM 5X-200, Doowon, Daejeon, Korea). A PMMA block (Vipi block GUM, Vipi, São Paulo, Brazil) of $98.5 \mathrm{~mm}$ in diameter and $25 \mathrm{~mm}$ in height was milled in wet condition with a minimum bur size of $1 \mathrm{~mm}$ while connectors were set to 10 (Fig. 4). For the RP method group, denture base resin (NextDent Base, NextDent, Soesterberg, Netherlands) was mixed during 1 hour using a stirring device (LC-3D Mixer, NextDent, Soesterberg, Netherlands) before printing process according to the manufacturers' instruction and checked for stable color of resin. Denture bases were vertically stacked with approved denture base material at a speed of 10 to $30 \mathrm{~mm} / \mathrm{h}$ using a digital light processing (DLP) 3D printer (Bio3D. W1, Bio3D Inc., Seoul, Korea). After completion of the printing process, denture bases were removed from the platform and cleaned with $99 \%$ isopropyl alcohol in an ultrasonic cleaner for 5 minutes followed by post curing for 15 minutes using an ultraviolet curing unit (LC-3D Print Box, NextDent, Soesterberg, Netherlands) (Fig. 5). After processing, denture bases of all groups were hydrated for 24 hours.

The left posterior border of the denture base was fixed 
with gypsum and coated with anti-glare spray (Cerec Optispray, Sirona dental systems, Bensheim, Germany). Casts and intaglio surfaces of denture bases were scanned with a model scanner (Rainbow scanner prime, Dentium, Suwon, Korea) and recorded as STL files. To determine the overall deformation of the base in the horizontal direction, the distance between 4 notches was measured. The most convex point of notches in the denture base and the most concave point of notches in the cast were used. Distance values between a-b, c-d, a-c, b-d, a-d, and b-c were measured with a surface matching software (Geomagic control $\mathrm{X}, 3 \mathrm{D}$ systems Inc., Rock-hill, SC, USA) (Fig. 6). Absolute values of the distance between the intaglio surface of the denture base and the cast were measured with the surface matching software. In this software, four notches of the denture base and the cast with an overlay guide were used to fit the denture base on the cast (Fig. 7). Two cross sections were made with the plane passing through the right and left

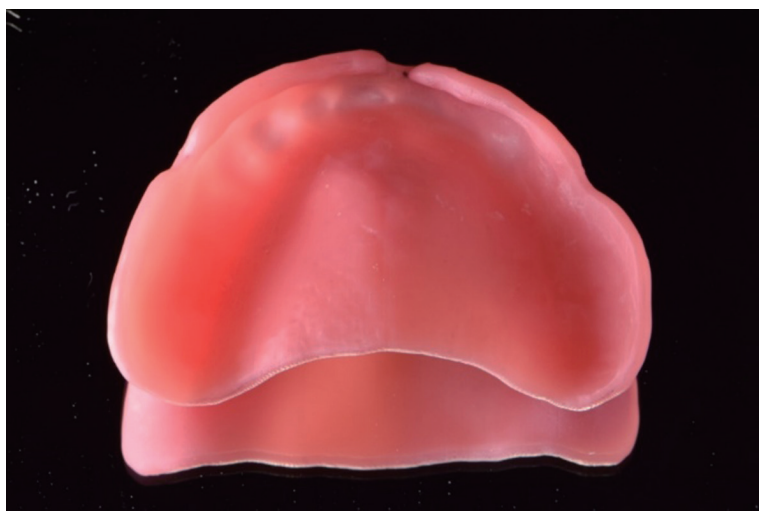

Fig. 5. Denture base fabricated by RP method.
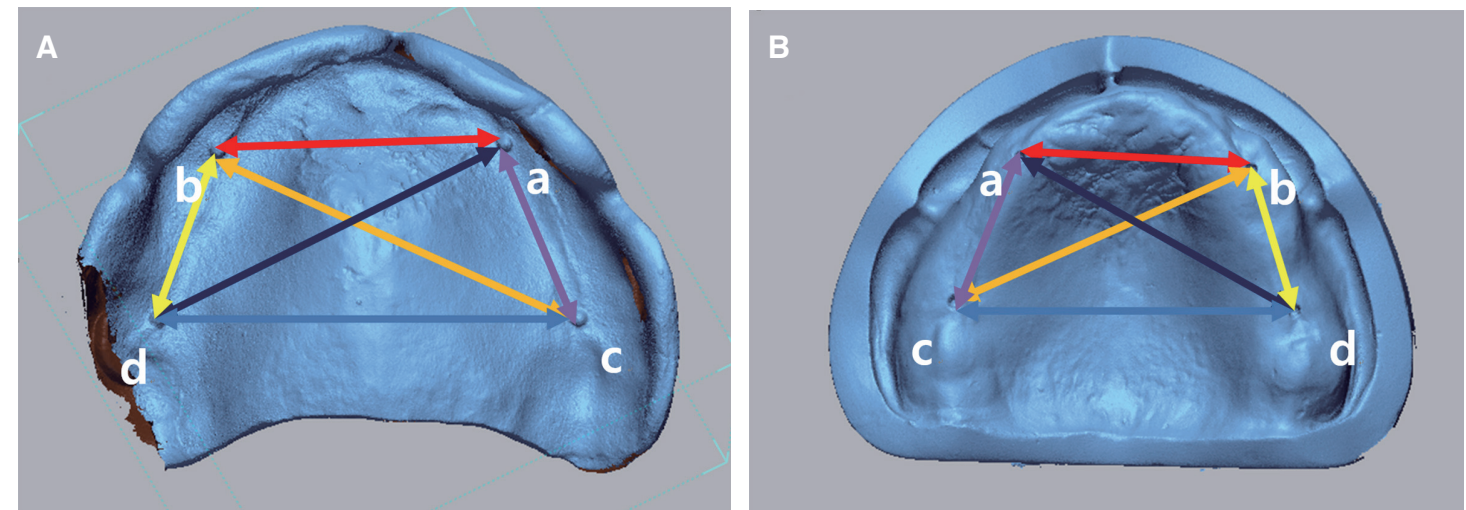

Fig. 6. Measurement of distance values between notches. (A) Point on scanned denture base, (B) Point on scanned cast, $(a, b)$ Right and left points crossing the anterior alveolar ridge and posterior alveolar ridges, (c, d) Right and left points on posterior alveolar ridge from $15 \mathrm{~mm}$ distance parallel to the midpalatal suture from right and left hamular notches. 

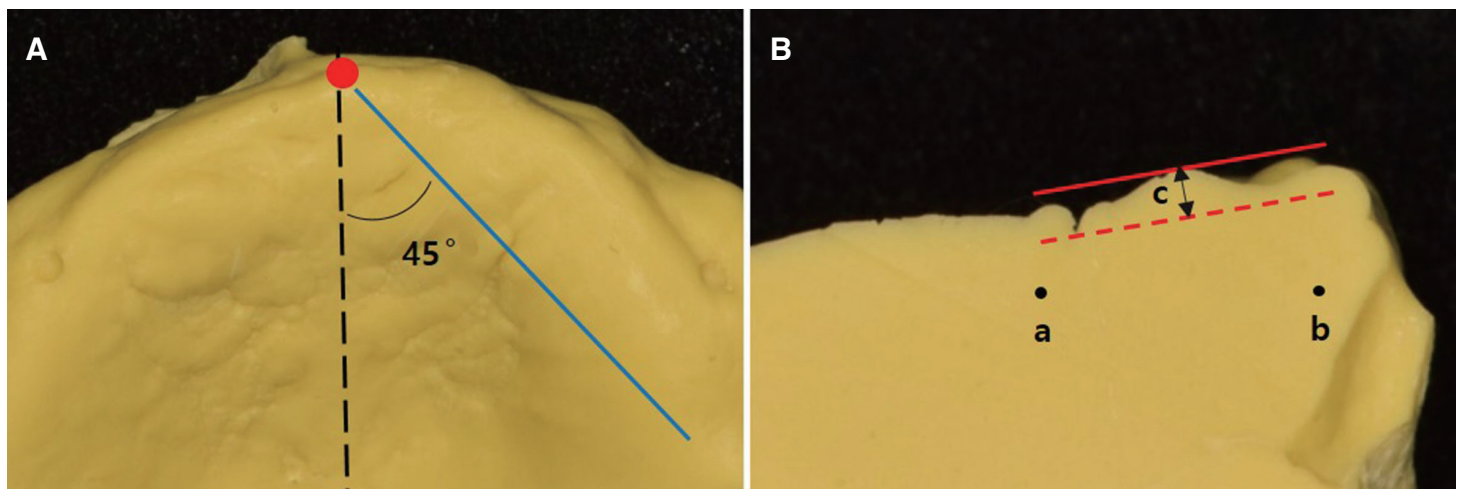

Fig. 8. Illustration of measurement method. (A) Occlusal view; the dotted line is the midpalatal suture and cutting the plane at a 45-degree angle from the most superior and anterior point of the midpalatal suture. The solid line is cutting line, (B) Cross-sectional view; points a and b are randomized points for ratio correction of photographs. Distance $\mathrm{c}$ is the distance between the most prominent point and the deepest point of the palatine rugae.

$2^{\text {nd }}$ upper premolar and the right and left $2^{\text {nd }}$ upper molar. Absolute values of the distance between the intaglio surface of the denture base were measured in each group at the intersection of two cross sections and the midpalatal suture. The palatine rugae resolution was evaluated by measuring the vertical distance between the most prominent point and the deepest point. The intaglio surface of the denture base in each group was impressed with polyvinylsiloxane impression materials (Imprint II Garant light body, 3M ESPE, St. Paul, MN, USA). From the forefront point of the midpalatal suture of the impression body, the plane was cut at an angle of $45^{\circ}$ with the midpalatal suture. The cross section was photographed by setting the camera at the shooting angle of $90^{\circ}$ with respect to the cross section. On the photograph, a line was drawn by connecting the upper part of one end and the upper part of the other end of the palatine rugae. Another line was drawn parallel to the line at the lowest point of the palatine rugae. The vertical distance between the most prominent point and this line was measured using Adobe Photoshop CC 2015 (Adobe Systems Inc., San Jose, CA, USA). Before photographing, two arbitrary points were displayed on the impression body. The ratio between the actual distance and the distance in the photograph was measured and applied to the vertical distance value in order to calculate the actual vertical distance value (Fig. 8). The entire measurement procedure was performed by one tester.

Results were analyzed using IBM SPSS Version 21.0 for WIN (SPSS Inc., Chicago, IL, USA). All data were subjected to normality and homogeneity of variance on a ShapiroWilk test for each group. One-way analysis of variance (ANOVA) with post hoc Tukey's honestly significant difference (HSD) was performed. The significance level was set at $5 \%$.

\section{RESULTS}

One-way ANOVA showed no significant $(P>.05)$ effect of a fabricating method on the overall deformation of the base in the horizontal direction, leading to the acceptance of the null hypothesis regarding the distance between 4 notches (Table 1). However, the null hypothesis for the fit accuracy in two points where the $2^{\text {nd }}$ upper premolar and the $2^{\text {nd }}$ upper molar crossed the midpalatal suture was rejected.

Table 1. Means and standard deviations (SDs) of distance values between each measurement point in the model and baseplates $(\mathrm{mm})$

\begin{tabular}{ccccccccccc}
\hline & \multicolumn{3}{c}{ Model } & \multicolumn{2}{c}{ Injection molding } & \multicolumn{3}{c}{ Milling } & \multicolumn{3}{c}{ Rapid prototyping } \\
& Mean & SD & Mean & SD & Mean & SD & Mean & SD \\
\hline a-b & 30.690 & 0.234 & 30.546 & 0.243 & 30.612 & 0.252 & 30.721 & 0.273 \\
C-d & 45.273 & 0.089 & 45.347 & 0.123 & 45.345 & 0.151 & 45.322 & 0.103 & .357 \\
a-c & 21.342 & 0.135 & 21.344 & 0.254 & 21.149 & 0.209 & 21.155 & 0.249 & .511 \\
b-d & 19.806 & 0.094 & 19.598 & 0.187 & 19.540 & 0.147 & 19.651 & 0.152 & .177 \\
a-d & 41.708 & 0.068 & 41.561 & 0.227 & 41.584 & 0.237 & 41.679 & 0.173 & .066 \\
b-c & 43.355 & 0.125 & 43.152 & 0.323 & 43.571 & 0.222 & 43.306 & 0.184 & .090 \\
\hline
\end{tabular}

*Significant difference at 0.05 level. 
There was no significant $(P=.94)$ interaction effect between the two factors of fabricating method and the region $\left(2^{\text {nd }}\right.$ upper premolar and the $2^{\text {nd }}$ upper molar) of the midpalatal suture (Fig. 9). There were statistically significant differences in the point where the $2^{\text {nd }}$ upper premolar crossed the midpalatal suture. The fit accuracy of the injection molding method was lower $(P<.05)$ than that of other methods (Table 2). At the point where the $2^{\text {nd }}$ upper molar crossed the midpalatal suture, the injection molding method also had significantly $(P<.05)$ lower fit accuracy than the other methods (Table 3). The mean value of discrepancies between the denture base and the cast in the midpalatal suture was found to be the lowest for those fabricated by the RP method, followed by those fabricated with the milling method and the injection molding method. The palatine rugae resolution was different by fabricating method. Milling and RP methods were similar while injection molding method had higher resolution (Fig. 10). The degree of the palatine rugae resolution was evaluated by measuring the vertical distance between the highest point and the lowest point. It was significantly $(P<.05)$ higher in the injection molding method than that in the milling or RP method (Table 4).

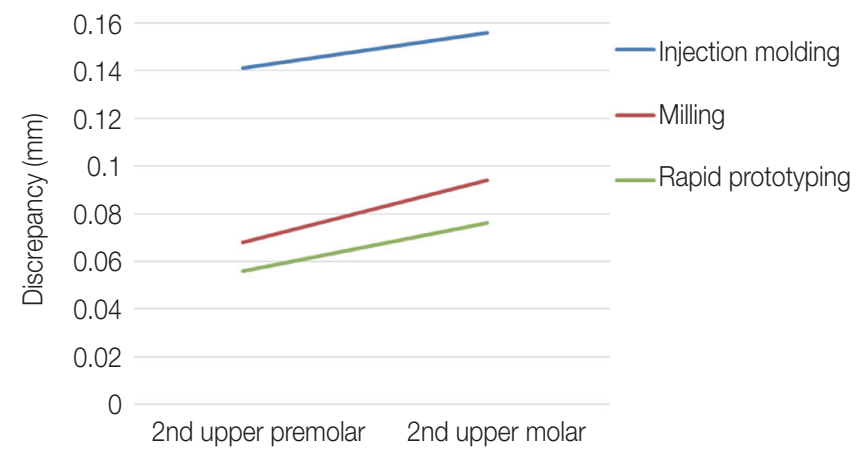

Fig. 9. Parallel tendency of each graph on the profile chart showing high possibility of no interaction effect between the fabricating method and the measurement point in the midpalatal suture.
Table 2. Means and SDs of discrepancy values between models and baseplates of dentures on the $2^{\text {nd }}$ upper premolar, crossing the midpalatal suture $(\mathrm{mm})$

\begin{tabular}{lll}
\hline & Mean & SD \\
\hline Injection molding & $0.141^{\mathrm{a}}$ & $0.058^{\mathrm{a}}$ \\
Milling & $0.068^{\mathrm{b}}$ & $0.037^{\mathrm{b}}$ \\
Rapid prototyping & $0.056^{\mathrm{b}}$ & $0.024^{\mathrm{b}}$ \\
\hline
\end{tabular}

Statistically significant difference found in the following: ${ }^{P}<<.05$ for milling and rapid prototyping; ${ }^{\mathrm{b} P}<.05$ for injection molding.

Table 3. Means and SDs of discrepancy values between models and baseplates of dentures on the $2^{\text {nd }}$ upper molar, crossing the midpalatal suture $(\mathrm{mm})$

\begin{tabular}{lll}
\hline & Mean & SD \\
\hline Injection molding & $0.156^{\mathrm{a}}$ & $0.080^{\mathrm{a}}$ \\
Milling & $0.094^{\mathrm{b}}$ & $0.040^{\mathrm{b}}$ \\
Rapid prototyping & $0.076^{\mathrm{b}}$ & $0.045^{\mathrm{b}}$ \\
\hline
\end{tabular}

Statistically significant difference found in the following: a $P<.05$ for milling and rapid prototyping; ${ }^{\mathrm{b} P}<.05$ for injection molding.

Table 4. Means and SDs of vertical distance values between the highest point and the lowest point of the palatine rugae $(\mathrm{mm})$

\begin{tabular}{lll}
\hline & Mean & SD \\
\hline Injection molding & $1.852^{\mathrm{a}}$ & $0.285^{\mathrm{a}}$ \\
Milling & $1.133^{\mathrm{b}}$ & $0.078^{\mathrm{b}}$ \\
Rapid prototyping & $1.206^{\mathrm{b}}$ & $0.171^{\mathrm{b}}$ \\
\hline
\end{tabular}

Statistically significant difference found in the following: ${ }^{P} P<.05$ for milling and rapid prototyping; ${ }^{\mathrm{b} P}<.05$ for injection molding.
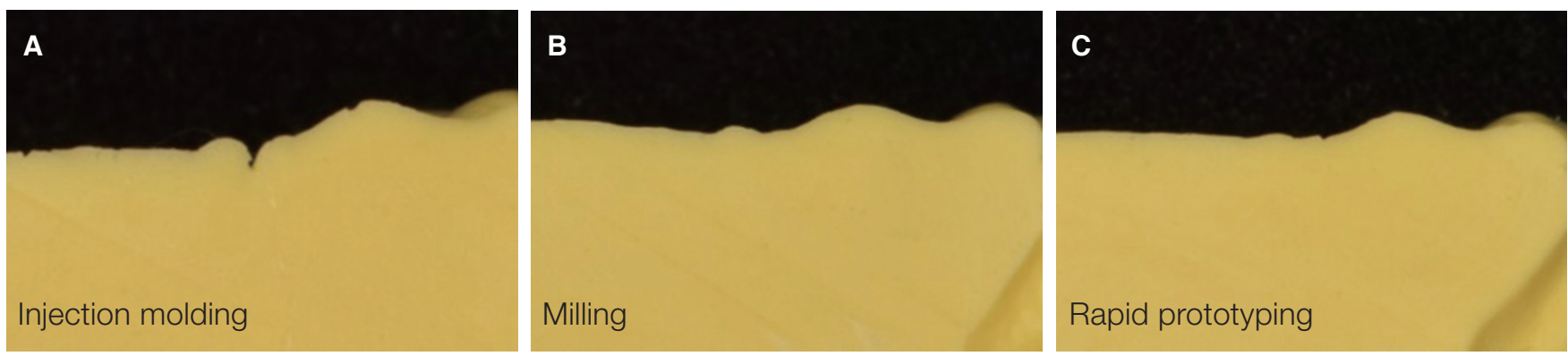

Fig. 10. Cross-sectional view of the palatine rugae reproduced with impression materials. (A) Injection molding method, (B) Milling method, (C) Rapid prototyping method. 


\section{DISCUSSION}

In all prostheses manufacturing, accuracy is the most important issue for clinicians. Shrinkage from polymerization has been inevitable since the introduction of polymer into the manufacturing of denture bases. For accuracy of prosthesis manufacturing, it is necessary to check the overall size and the fit of the intaglio surface.

There was no statistical significance in the amount of deformation of the base in the horizontal direction through the measurement of the distances between notches on the ridge. There was no difference in the accuracy of the overall size for each method, and the RP method, which has not been studied much, has not disclosed much deformation when making the complete denture base. There was no difference in the accuracy of the overall size for each method.

According to studies on fabricating methods for maxillary complete denture, the misfit of the base varies depending on the region. ${ }^{21}$ The gap between the denture base and the cast is generally influenced by shrinkage of the resin material during polymerization with a tendency of the internal stress toward the central region of the denture base and subsequent distortion caused by limitation due to the surface shape of the alveolar ridge. ${ }^{22}$ Therefore, in the central area of the posterior border and buccal vestibule area, the largest gap is generally measured when close contact state was maintained around the ridge crest. ${ }^{23}$ In the present study, the discrepancy was measured on the $2^{\text {nd }}$ upper premolar, the center of anteroposterior distance, the $2^{\text {nd }}$ upper molar, posterior region, points crossing, and the midpalatal suture.

Recently, many studies have compared the accuracy between CAD/CAM milling and conventional methods. According to the study of Goodacre et al., ${ }^{18}$ the CAD/CAM milling method showed less misfit than the injection molding method in the maxillary complete denture, including the edge of denture border, $6 \mathrm{~mm}$ away from denture border, palate, posterior palatal seal area, and crest of alveolar ridge. Steinmassl et al..$^{24}$ have compared the congruence of the compression molding method and various CAD/CAM milling systems in various regions of the maxillary complete denture. CAD/CAM milling systems had higher congruence with denture-bearing tissues than compression molding methods. In conventional and almost all CAD/CAM systems, alveolar ridge and palate is the most accurate fit region, and posterior palatal seal and anterior and lateral seal regions showed the largest extent of misfit. ${ }^{24}$

In injection molding method, as mentioned in many previous researches, the misfit due to the polymerization shrinkage and the internal stress after the polymerization is relatively high in the midpalatal suture area because of the shape and the position. While conventional methods using PMMA should consider polymerization shrinkage, the milling method is processed by subtraction of an industrially pre-polymerized PMMA blank (puck) that has final dimension. There is no dimensional deformation of the denture base, theoretically. Thus, milling is the most reproducible technique. In the present study, the congruence of the base fabricated by the milling method was mainly influenced by the accuracy of the scanner used for the measurement and the precision of the milling process. Two types of model scanners were used; D800 model scanner was used to scan mounted casts for designing denture and Rainbow scanner prime was used to scan the manufactured denture bases of each group. Each of the two procedures was independent and the results was measured and analyzed from scanning with same model scanner, Rainbow scanner prime. According to the manufacturer, Rainbow scanner prime has accuracy within $10 \mu \mathrm{m}$. Since the amount of the misfit was larger than the scanner's accuracy, the experimental setup and measurement in this study appeared to be appropriate for measuring differences. An additional error could be caused by the powder that coated the denture base for scanning. Schaefer et al. ${ }^{25}$ have addressed that marginal fit and internal adaptation in partial coverage restorations were adversely effected by the powder for scanning. However, in the same report, deviations were within clinically acceptable thresholds. In vitro studies of coating powder have shown that surface pre-treatment with powder does not significantly decreased the scanning accuracy ${ }^{26}$ because of small particle size of aerosol sprays around $5 \mu \mathrm{m} .{ }^{25}$ Although this error may be considered in the field of fixed prosthodontics and inaccuracies of degree of micrometer are considered clinically acceptable in the field of complete denture prosthodontics, this hypothesis must be scientifically proven. Considering the reason of additional misfit, the milling process can be affected by a milling bur, the characteristic of the area to be milled, and the machining axis. Steinmassl et al. ${ }^{24}$ have reported that the major challenge in the milling method may be reproducing undercut regions, based on their finding that all CAD/CAM denture systems have some problems in reproducing the anterior and lateral denture border area that often include undercut regions below the alveolar crest.

Stereolithography is one of RP methods. The advantage of this technology is flexibility due to the range of available machines, low percentage of wasted raw material, and ability to print complex geometries. The main problems of printing method are staircase effect, low reproducibility, and the necessity of supporting structures. These supporting structures need additional material and time. ${ }^{27}$ The main difference between stereolithography and DLP is the light source. Arc lamp or digital micromirror device and small micromirrors attached in a matrix on a semiconductor chip create the image in DLP. Each micromirror reflects one or more pixels in the projected image. The resolution of the image is proportional to the number of micromirrors. ${ }^{28}$ Discrepancies of the RP method can be incorporated in each step, including designing in the CAD software, slicing procedure in the printing software, and printing. The accuracy of printed object is influenced by many various factors such as light intensity, direction and angle of printing, ${ }^{29,30}$ number of layers, ${ }^{31}$ software, ${ }^{32}$ shrinkage between layers, ${ }^{30}$ amount of supporting structure, ${ }^{29}$ and post processing pro- 
cedures. Each material has its own activation range, wavelength, intensity, and exposure time in each 3D printer and not all printing materials are compatible with all $3 \mathrm{D}$ printers. Because of disparities in protocol (selected technology, parameters of printers, and the material used for printing), it is very difficult to compare the results of various studies. ${ }^{33}$

In the RP method group, discrepancy values in the palatal region $\left(0.056 \pm 0.024 \mathrm{~mm}\right.$ on $2^{\text {nd }}$ upper premolar, and $0.076 \pm 0.045 \mathrm{~mm}$ on $2^{\text {nd }}$ upper molar) were very low. There are few studies on the fit of the denture base fabricated by the RP method. It is currently unknown in exactly which process the error occurs due to complexity of the printing process and uncontrolled recoveries of duplicated errors. However, the possibility of using the RP method with considerable accuracy was confirmed through the present study.

Light-cured acrylic resins for fabricating the denture base are available on the 3D printer. There are a few products licensed for use in patients. The NextDent Base used in this study is FDA approved. The safety of these RP acrylic materials is being tested and these materials are being assessed for long-term use. In this base material, post curing is done for 30 minutes under certain conditions following the instructions for use. However, in this study, 15 minutes was performed with different polymerization conditions in order to correct the accuracy. In the case of less polymerization, the leakage of residual monomers or decreasing strength and the color of the fabricated denture may be insufficient. However, there was sufficient polymerization in the confirmation of the denture base color in this study.

There was a high possibility of no interaction effect between the fabricating method and the measurement point in the midpalatal suture through parallel tendency of not crossing each graph on the profile chart (Fig. 9). Generally, there is a difference in the misfit for each method depending on the region due to characteristics of the fabricating method. However, effects by each fabricating method on two points measured in this study were almost equal. According to McLaughlin et al., the effect of the palate depth (palatal depth of $12 \mathrm{~mm}$ and $18 \mathrm{~mm}$ classified by Johnson et al. ${ }^{20}$ ) on the misfit by manufacturing method (conventional method and CAD/CAM milling method) was not observed. ${ }^{19}$ In the present study, the palatal depth of the cast was $12 \mathrm{~mm}$ and the palatal depth did not influence the results.

The misfit in conventional measuring method is determined by measuring the actual misfit after cutting ${ }^{21}$ or using a device such as computed tomography after fitting the denture on the cast. ${ }^{23}$ The development of CAD software makes it possible to easily identify the cross-section of the desired region of the program. The measurement of the misfit using the CAD software sometimes displays a negative value, which is different from the conventional method because the denture base image is fitted to the cast based on the reference points. In this study, statistical analysis was performed for absolute values of the measurement to eliminate errors due to negative values. Because this method is different from fitting the denture to the patient, it is difficult to interpret clinically. However, it is more meaningful in measuring the accuracy of the fabricated denture itself. By measuring the discrepancy, it is possible to improve the convenience and perfection of the clinical procedure by fabricating more accurate dentures and predicting areas where errors may occur.

In comparison with the degree of palatine rugae resolution of the intaglio surface of the base, the injection molding method was found to be the most precise method. The accuracy of the 3 Shape D800 scanner was $7-8 \mu \mathrm{m}$. When light was used for scanning, specific areas in the palatine rugae of the cast might not have been scanned. In the milling method, the size of milling bar and undercuts for the axis of milling bar are limited. The intaglio surface of a $\mathrm{CAD} / \mathrm{CAM}$ milled denture is not as smooth as the intaglio surface of the base fabricated by conventional method and is rather layered than conventional method. Goodacre et al's study also has shown similar texture on the intaglio surface images. ${ }^{18,34}$ This surface shape is inevitable because milling bar is larger than the size of stone particle. The resolution of the Bio3D. W11 DLP 3D printer used in this study was $57 \mu \mathrm{m}$ and 50 to $100 \mu \mathrm{m}$ for the one layer. The resolution is the smallest or the finest degree that could be reproduced by the $3 \mathrm{D}$ printer and it is specific for each $3 \mathrm{D}$ printer. The resolution should be defined in $\mu \mathrm{m}$ or dots per inch (dpi) for each $\mathrm{x}, \mathrm{y}$, and z-axis and generally, the z-axis corresponds to the thickness of the layer. ${ }^{35}$ These resolutions can be confirmed simply by the shape, but in this study, the vertical distance between the highest point and the lowest point was measured and quantified.

The limitation of this study includes difficulty in confirming the error in each stage of the $3 \mathrm{D}$ printing procedure because of complicating factors. Future research is needed to confirm the error in each stage and overcome it. Clinicians who are concerned with the CAD/CAM milling and $3 \mathrm{D}$ printing technique for fabricating a complete denture may obtain clinically acceptable fit accuracy and retention. With generally equivalent fit accuracy between the milling and RP methods of the CAD/CAM work flow, other factors such as cost, mechanical property, risk of the residual monomer, color stability, ease of fabrication, and clinical application might need to be considered. Future studies should evaluate these factors in order to use CAD/ CAM technology more stably and accurately.

\section{CONCLUSION}

There was no significant difference in the overall deformation in the horizontal direction among the three methods through comparison of distances between the 4 notches of the ridge. Comparison of fit accuracy between the cast and the maxillary complete denture base was evaluated on the $2^{\text {nd }}$ upper premolar and the $2^{\text {nd }}$ upper molar crossing the midpalatal suture, showing relatively high deformation in the conventional method due to polymerization shrinkage and the internal stress. The mean value of discrepancies was the lowest in the RP method, followed by that in the milling 
method and the injection molding method. The injection molding method had significantly lower fit accuracy than the other two CAD/CAM methods at two points. The degree of resolution was evaluated by measuring the vertical distance between the highest point and the lowest point in the palatine rugae area because of the complexity of the architecture. The injection molding method had significantly higher resolution than the milling method and the RP method.

\section{ORCID}

Suji Lee https://orcid.org/0000-0002-3325-1426

Seoung-Jin Hong https://orcid.org/0000-0002-7460-8487

Janghyun Paek https://orcid.org/0000-0002-1286-3140

Ahran Pae bttps://orcid.org/0000-0001-8758-0754

Kung-Rock Kwon https://orcid.org/0000-0002-9777-8980

Kwantae Noh bttps://orcid.org/0000-0003-3480-7737

\section{REFERENCES}

1. Kawara M, Komiyama O, Kimoto S, Kobayashi N, Kobayashi K, Nemoto K. Distortion behavior of heat-activated acrylic denture-base resin in conventional and long, low-temperature processing methods. J Dent Res 1998;77:1446-53.

2. Parvizi A, Lindquist T, Schneider R, Williamson D, Boyer D, Dawson DV. Comparison of the dimensional accuracy of injection-molded denture base materials to that of conventional pressure-pack acrylic resin. J Prosthodont 2004;13:83-9.

3. Darvell BW, Clark RK. The physical mechanisms of complete denture retention. Br Dent J 2000;189:248-52.

4. Jacob RF. The traditional therapeutic paradigm: complete denture therapy. J Prosthet Dent 1998;79:6-13.

5. Jacobson TE, Krol AJ. A contemporary review of the factors involved in complete denture retention, stability, and support. Part I: retention. J Prosthet Dent 1983;49:5-15.

6. Jacobson TE, Krol AJ. A contemporary review of the factors involved in complete dentures. Part II: stability. J Prosthet Dent 1983;49:165-72.

7. Jacobson TE, Krol AJ. A contemporary review of the factors involved in complete dentures. Part III: support. J Prosthet Dent 1983;49:306-13.

8. Pryor WJ. Injection molding of plastics for dentures. J Am Dent Assoc 1942;29:1400-8.

9. Huggett R, Zissis A, Harrison A, Dennis A. Dimensional accuracy and stability of acrylic resin denture bases. J Prosthet Dent 1992;68:634-40.

10. Goodacre CJ, Garbacea A, Naylor WP, Daher T, Marchack CB, Lowry J. CAD/CAM fabricated complete dentures: concepts and clinical methods of obtaining required morphological data. J Prosthet Dent 2012;107:34-46.

11. Bidra AS, Taylor TD, Agar JR. Computer-aided technology for fabricating complete dentures: systematic review of historical background, current status, and future perspectives. J Prosthet Dent 2013;109:361-6.

12. Infante L, Yilmaz B, McGlumphy E, Finger I. Fabricating complete dentures with CAD/CAM technology. J Prosthet Dent 2014;111:351-5.
13. Maeda Y, Minoura M, Tsutsumi S, Okada M, Nokubi T. A CAD/CAM system for removable denture. Part I: Fabrication of complete dentures. Int J Prosthodont 1994;7:17-21.

14. Matsuda T, Goto T, Yagi K, Kashiwabara T, Ichikawa T. Partdigitizing system of impression and interocclusal record for complete denture fabrication. J Prosthodont 2016;25:503-9.

15. Kuesel AC, Graschew G, Hull WE, Lorenz W, Thielmann HW. 31P NMR studies of cultured human tumor cells. Influence of $\mathrm{pH}$ on phospholipid metabolite levels and the detection of cytidine 5'-diphosphate choline. NMR Biomed 1990;3:78-89.

16. Wimmer T, Gallus K, Eichberger M, Stawarczyk B. Complete denture fabrication supported by CAD/CAM. J Prosthet Dent 2016;115:541-6.

17. Munari LS, Cornacchia TP, Moreira AN, Gonçalves JB, De Las Casas EB, Magalhães CS. Stress distribution in a premolar 3D model with anisotropic and isotropic enamel. Med Biol Eng Comput 2015;53:751-8.

18. Goodacre BJ, Goodacre CJ, Baba NZ, Kattadiyil MT. Comparison of denture base adaptation between CAD-CAM and conventional fabrication techniques. J Prosthet Dent 2016;116:249-56.

19. McLaughlin JB, Ramos V Jr, Dickinson DP. Comparison of fit of dentures fabricated by traditional techniques versus CAD/CAM technology. J Prosthodont 2017 Nov 14.

20. Johnson DL, Holt RA, Duncanson MG Jr. Contours of the edentulous palate. J Am Dent Assoc 1986;113:35-40.

21. Sykora O, Sutow EJ. Posterior palatal seal adaptation: influence of processing technique, palate shape and immersion. J Oral Rehabil 1993;20:19-31.

22. Johnson DL, Duncanson MG Jr. The plastic postpalatal denture seal. Quintessence Int 1987;18:457-62.

23. Lee CJ, Bok SB, Bae JY, Lee HH. Comparative adaptation accuracy of acrylic denture bases evaluated by two different methods. Dent Mater J 2010;29:411-7.

24. Steinmassl O, Dumfahrt H, Grunert I, Steinmassl PA. CAD/ CAM produces dentures with improved fit. Clin Oral Investig 2018;22:2829-35.

25. Schaefer O, Decker M, Wittstock F, Kuepper H, Guentsch A. Impact of digital impression techniques on the adaption of ceramic partial crowns in vitro. J Dent 2014;42:677-83.

26. Ender A, Mehl A. Influence of scanning strategies on the accuracy of digital intraoral scanning systems. Int J Comput Dent 2013;16:11-21.

27. Petrovic V, Gonzalez JVH, Ferrando OJ, Gordillo JD, Puchades JRB, Griñan LP. Additive layered manufacturing: sectors of industrial application shown through case studies. Int J Prod Res 2011;49:1061-79.

28. Groth C, Kravitz ND, Jones PE, Graham JW, Redmond WR. Three-dimensional printing technology. J Clin Orthod 2014;48:475-85.

29. Alharbi N, Osman R, Wismeijer D. Effects of build direction on the mechanical properties of 3D-printed complete coverage interim dental restorations. J Prosthet Dent 2016;115:760-7.

30. Braian M, Jimbo R, Wennerberg A. Production tolerance of additive manufactured polymeric objects for clinical applications. Dent Mater 2016;32:853-61. 
31. Allen S, Dutta D. On the computation of part orientation using support structures in layered manufacturing. Proceedings of the International Solid Freeform Fabrication Symposium; Austin, TX, 1994. p. 259-69.

32. Plooij JM, Maal TJ, Haers P, Borstlap WA, Kuijpers-Jagtman AM, Bergé SJ. Digital three-dimensional image fusion processes for planning and evaluating orthodontics and orthognathic surgery. A systematic review. Int J Oral Maxillofac Surg 2011;40:341-52.

33. Revilla-León M, Özcan M. Additive manufacturing technologies used for processing polymers: Current status and potential application in prosthetic dentistry. J Prosthodont 2018 Apr 22.

34. Srinivasan M, Cantin Y, Mehl A, Gjengedal H, Müller F, Schimmel M. CAD/CAM milled removable complete dentures: an in vitro evaluation of trueness. Clin Oral Investig 2017;21:2007-19.

35. Puebla K, Arcaute K, Quintana R, Wicker RB. Effects of environmental conditions, aging, and build orientations on the mechanical properties of ASTM type I specimens manufactured via stereolithography. Rapid Prototyp J 2012;18:374-88. 